\title{
Lemierre syndrome: early recognition and management
}

\author{
Chani Tromop-van Dalen MBChB, Ann-Marie Mekhail MBChB
}

1 previously well 16-year-old boy presented to the emergency department with fever, rigor, and pain in his chest, neck and dorsal right hand. The illness had begun 10 days earlier with a sore throat, which was reviewed at an after-hours walk-in clinic and presumed to be viral. He remained unwell and began to experience neck pain, and so presented to the emergency department two days later. Following a normal lumbar puncture, he was discharged home with a provisional diagnosis of Epstein-Barr virus. A throat swab was taken during this visit that grew group G Streptococcus. Treatment was not given as per hospital policy for this type of infection.

Two days later he returned to the emergency department with persistent fever, rigor, neck pain and pleuritic chest pain. The sore throat had resolved, but his right hand had become swollen and painful.

The patient appeared clammy and pale despite normal vital signs. His neck was tender to the left of the trachea, along the line of the sternocleidomastoid; there were no signs of meningism. His throat appeared inflamed, but there was no tonsillar exudate. His cervical lymph nodes were tender and enlarged. There were no rashes or peripheral stigmata of infective endocarditis. Cardiorespiratory and abdominal examination were normal. The dorsum of the right hand was tender and swollen, and had reduced range of motion secondary to pain.

Initial investigations showed an inflammatory response, with C-reactive protein 106 (normal 0-5) $\mathrm{mg} / \mathrm{L}$ and leukocyte count 13.8 (normal 4.0-11.0) $\times 10^{9} / \mathrm{L}$. Liver tranaminase levels were mildly elevated, the international normalized ratio was 1.3 and the albumin level was 23 (normal 34-48) g/L. Renal function and electrolytes were normal. A chest radiograph showed numerous right-sided pulmonary nodules. The right wrist joint was aspirated, but no fluid was obtained. Computed tomography of the neck and chest showed abnormal filling of contrast in the left internal jugular vein extending from C3 to the thoracic inlet (Figure 1); there were no drainable abscesses in the neck. There were multiple cavitating lesions within both lungs and a small associated empyema (Figure 2).

The patient was given empirical treatment with intravenous piperacillin-tazobactam and clindamycin based on a radiologic diagnosis of Lemierre syndrome, pending the results of cultures and sensitivities. He was transferred to the high dependency (intermediate care) unit because of severe sepsis and low urine output. Blood cultures later grew Fusobacterium necrophorum.

Despite treatment, the patient continued to have temperature spikes and his right hand became progressively more painful and swollen. Ultrasonography confirmed a superficial collection in the soft tissue of the dorsum of the right hand, which required two surgical washouts and drain insertion. Ultrasonography of the neck, performed to investigate increasing neck swelling, showed that the thrombus had extended to completely occlude the left internal jugular vein. Anticoagulation with warfarin, with bridging enoxaparin, was initiated on advice from the hematology team. His temperatures returned to normal following these further treatments.

The patient improved and was discharged home after 13 days on intravenous ceftriaxone and metronidazole. One week after discharge, he had fully recovered, and treatment was changed to oral metronidazole monotherapy for a further three weeks and a total of three months' anticoagulation treatment.

\section{KEY POINTS}

- The classic presentation of Lemierre syndrome is that of a young (age 15-24 yr) patient with a recent oropharyngeal infection presenting with sepsis, unilateral neck pain from internal jugular thrombophlebitis, and clinical or radiologic evidence of septic emboli.

- Isolation of Fusobacterium species should prompt the clinician to consider a diagnosis of Lemierre syndrome.

- The mainstay of treatment for Lemierre syndrome is administration of intravenous antibiotics; empiric treatment should cover anaerobic organisms, Streptococcus and Staphylococcus species, and include a $\beta$-lactamase inhibitor, such as piperacillin-tazobactam. 


\section{Discussion}

In 1936, Lemierre published a series of 20 cases of "anaerobic post-anginal sepsis." ${ }^{1}$ The illness consisted of a history of oropharyngeal infection followed by anaerobic sepsis with Bacillus funduliformis (now known as F. necrophorum) and thrombus of the internal jugular vein. ${ }^{1}$ Today, it is a rare but life-threatening disease. In the preantibiotic era, this disease had a fulminant and often fatal progression within two weeks. ${ }^{2}$ With the advent of antibiotics, mortality has been reported as low as $5 \%$. About $10 \%$ of patients experience permanent sequelae, ${ }^{3}$ including longterm neurologic effects of cerebral abscesses and meningitis, valve replacements for endocarditis and chronic osteomyelitis. ${ }^{4}$

\section{Diagnosis}

Following the initial oropharyngeal infection, a parapharyngeal abscess forms and thrombophlebitis of the internal jugular vein occurs. The most common symptoms at the beginning are sore throat $(33 \%)$, neck mass $(23 \%)$ and neck pain $(20 \%){ }^{5}$ Presentation with subsequent complications does not tend to occur until one to three weeks later. ${ }^{4,6,7}$

Riordan ${ }^{8}$ proposed a definition of the syndrome with three criteria: history of recent upper

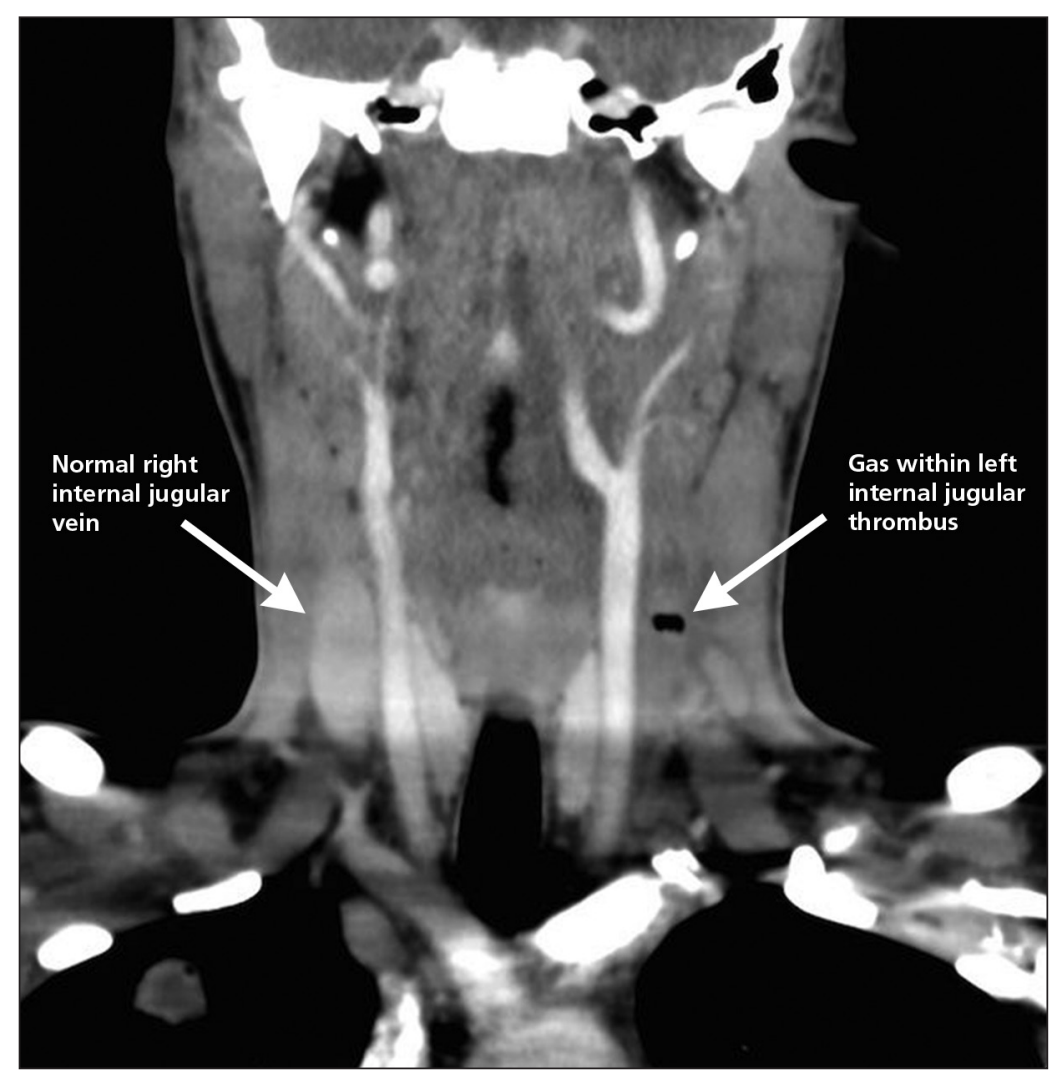

Figure 1: Computed tomographic image of the neck of a 16-year-old boy showing left thrombosed internal jugular vein and gas within the thrombus. respiratory tract infection or compatible clinical findings; evidence of metastatic infectious lesions; and either evidence of internal jugular vein thrombophlebitis or isolations of $F$. necrophorum or Fusobacterium species from blood culture or a normally sterile site. Therefore, the diagnosis can be made based on clinical and radiologic findings in the absence of culture results.

The incidence is about 0.6-3.6 cases per million overall, ${ }^{6,7}$ however, incidence rates appear to be increasing. ${ }^{2,6}$ This may be a result of increased reporting or improved identification of cases with more advanced imaging techniques. It has also been hypothesized that the increasing incidence may be due to reduced antibiotic prescribing for sore throats in primary practice because of concerns about development of antibiotic resistance. ${ }^{2,6}$

Incidence rates rise to 14.4 cases per million in those aged $15-24$ years. ${ }^{3,7}$ This rise is thought to be due to involution of tonsillar tissue, predisposing this age group to infections in tonsillar crypts. ${ }^{4}$

Complications of Lemierre syndrome are a result of septic emboli from the thrombus, systemic effects of sepsis and clot extension. Sites of metastatic infection, in order of frequency of involvement, are lung, joint, muscle, bone, liver, skin, spleen and endocardium. ${ }^{8}$ Pleuropulmonary involvement occurs in $97 \%$ of cases. ${ }^{6,7}$

The most commonly implicated organism is $F$. necrophorum, a gram-negative anaerobic bacillus. ${ }^{2,8}$ Unlike other anaerobic infections, which are frequently polymicrobial, it is often the sole isolate from blood cultures. ${ }^{2}$ It produces hemagglutinin, promoting platelet aggregation, which has been put forward as the explanation for thrombus formation. ${ }^{4,5,9}$ Detection of this organism should prompt clinicians to investigate for internal jugular vein thrombus; however, given the time required for anaerobic blood culture results, the diagnosis of Lemierre syndrome is first and foremost clinical.

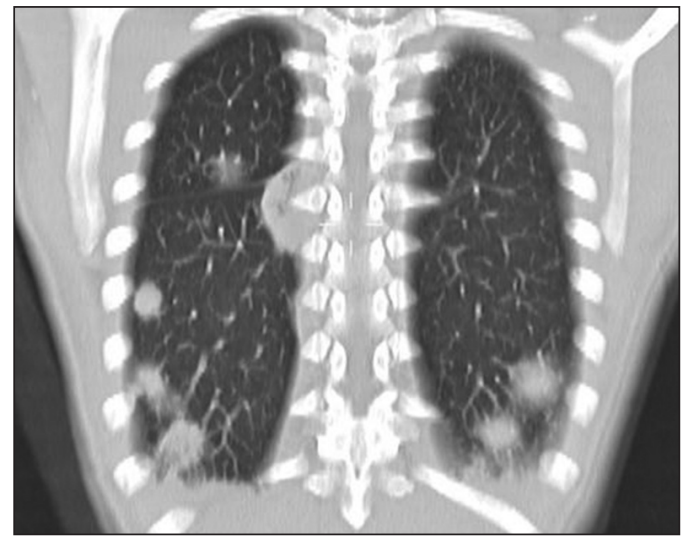

Figure 2: Computed tomographic image of the thorax showing multiple septic pulmonary emboli. 


\section{Treatment}

Fusobacterium necrophorum is usually susceptible to penicillin, clindamycin and metronidazole, whereas it tends to be resistant to macrolides. ${ }^{2}$ Antimicrobial combinations resistant to $\beta$-lactamase, such as piperacillin-tazobactam, are also recommended, because up to a quarter of $F$. necrophorum can be $\beta$-lactamase producing. ${ }^{2,7,8}$ Other bacteria that have been identified include Streptococcus and Staphylococcus species, and empiric therapy must also cover these organisms. ${ }^{6,7}$ The mainstay of treatment for Lemierre syndrome is early administration of intravenous antibiotics and surgical drainage of collections. Prolonged therapy for three to six weeks is recommended to allow time for antibiotics to penetrate into the fibrin clot and necrotic abscesses. ${ }^{7}$

Owing to the rarity of the disease, the evidence for use of anticoagulation to break down the internal jugular vein thrombus is limited to anecdotal case observations. ${ }^{7}$ In our case, anticoagulation was started because of extension of the thrombus, soon after surgical drainage of the soft tissue collection on the dorsum of the hand. Because anticoagulation occurred within days of surgery, it is difficult to assess whether the addition of this treatment was a factor in clinical improvement.

\section{Early detection}

Fusobacterium necrophorum appears to be responsible for $10 \%$ of all throat infections, ${ }^{5}$ but early detection on clinical grounds is challenging because of the wide differential diagnosis for sore throat. The differential diagnosis includes viral pharyngitis, Epstein-Barr virus, streptococcal pharyngitis, peritonsillar abscess and retropharyngeal abscess. In unvaccinated patients, diphtheria and epiglottitis should also be considered. Infection with $F$. necrophorum is more likely in patients aged 15-24 years, those with recurrent sore throats and those with a raised C-reactive protein level. ${ }^{3,5,6}$ However, it is appreciated that these features are not unique to this bacteria.

In our patient's case, a throat swab was taken during his first visit to the emergency department. The culture grew group G Streptococcus; this infection was not treated, because the hospital's policy is to treat only group A Streptococcus infection, the primary culprit in rheumatic fever. Culturing Fusobacterium, however, requires a special medium, which our laboratory does not routinely use unless requested to do so. As a result, the throat culture in our patient would not have shown Fusobacterium.
This raises the question of the utility of testing for Fusobacterium in those with throat infections. The reasoning for testing for fusobacterial throat infections is similar to that of testing for group A streptococcal throat infections that can cause the rare, but severe, complication of rheumatic fever. Bank and colleagues ${ }^{3}$ conducted a detailed cost-effectiveness analysis of testing cases of pharyngitis in the high-risk age group (15-24 yr) with throat swabs to detect $F$. necrophorum and treatment of positive results with antibiotics. They found that, because of the high cost burden of Lemierre syndrome and peritonsillar abscess, it would take only a few cases to be prevented for testing for Fusobacterium to be cost-effective. ${ }^{3}$

Although it is plausible that giving treatment for throat cultures positive for $F$. necrophorum would lead to a reduction in incidence of Lemierre syndrome, this requires further investigation with a prospective study. If this were deemed to be an effective public health measure, throat swabs would need to be taken with the initial sore throat, because once thrombophlebitis has occurred, the infection is largely intravascular and there is rarely any pharyngeal exudate. $^{7}$

\section{References}

1. Lemierre A. On certain septicaemias due to anaerobic organisms. Lancet 1936;701-3.

2. Golpe R, Marín B, Alonso M. Lemierre's syndrome (necrobacillosis). Postgrad Med J 1999;75:141-4.

3. Bank S, Christensen K, Kristensen LH, et al. A cost-effectiveness analysis of identifying Fusobacterium necrophorum in throat swabs followed by antibiotic treatment to reduce the incidence of Lemierre's syndrome and peritonsillar abscesses. Eur J Clin Microbiol Infect Dis 2013;32:71-8.

4. Chan ED, Hermanoff MH, Connick E. Lemierre's syndrome: review of the English literature over the Past 25 years. Clin Pulm Med 1999;6:333-8.

5. Karkos PD, Asrani S, Karkos CD, et al. Lemierre's syndrome: a systematic review. Laryngoscope 2009;119:1552-9.

6. Asnani J, Jones S. Case review. Lemierre's syndrome. J Fam Pract 2014;63:193-6.

7. Kuppalli K, Livorsi D, Talati NJ, et al. Lemierre's syndrome due to Fusobacterium necrophorum. Lancet Infect Dis 2012;12: 808-15.

8. Riordan T. Human infection with Fusobacterium necrophorum (Necrobacillosis), with a focus on Lemierre's syndrome. Clin Microbiol Rev 2007;20:622-59.

9. Moore BA, Dekle C, Werkhaven J. Bilateral Lemierre's syndrome: a case report and literature review. Ear Nose Throat $J$ 2002;81:234-6, 238-40, 242.

Affiliations: Capital \& Coast District Health Board and Hutt Valley District Health Board, Wellington, New Zealand

Contributors: Both authors wrote the article, approved the final version and agreed to act as guarantors of the work.

Acknowledgements: The authors thank Dr. Shyh Poh Teo, Dr. Matthew Kelly and Dr. Ken Bricker for their assistance with preparing the article and images. 